CHANGES IN STRENGTH, POWER AND SPEED ACROSS A SEASON IN ENGLISH COUNTY CRICKETERS

\begin{tabular}{|r|l|}
\hline Journal: & International Journal of Sports Physiology and Performance \\
\hline Manuscript ID & IJSPP.2015-0524.R2 \\
\hline Manuscript Type: & Original Investigation \\
\hline Keywords: & competition, maintenance, performance, jump, sprint, squat \\
\hline \multicolumn{2}{|c}{} \\
\hline
\end{tabular}

\title{
SCHOLARONE ${ }^{\text {M }}$ \\ Manuscripts
}




\section{CHANGES IN STRENGTH, POWER AND SPEED ACROSS A SEASON IN ENGLISH COUNTY CRICKETERS}

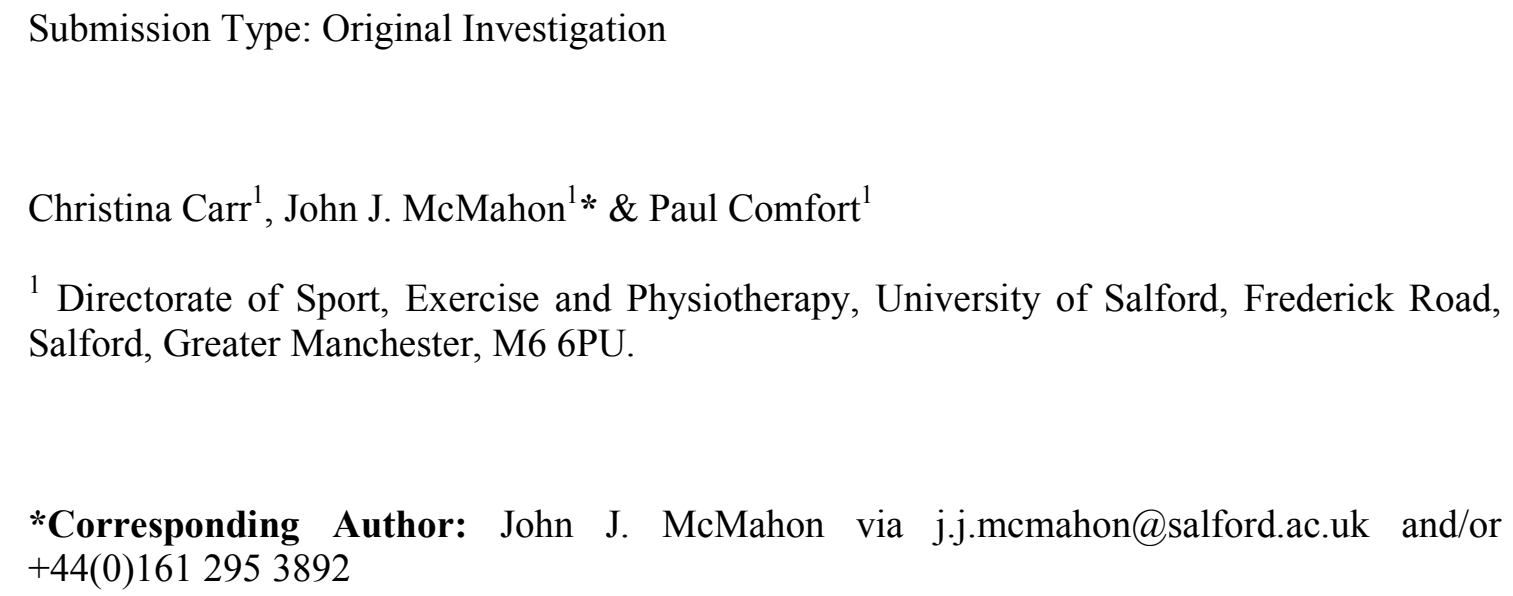

Preferred Running Head: Seasonal Variations in Strength, Power and Speed

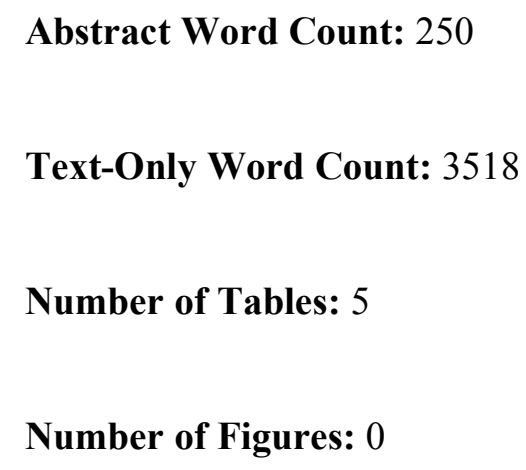




\begin{abstract}
Purpose: Previous research has investigated changes in athletes' strength, power and speed performances across the competitive season of many sports, although this has not been explored in cricketers. The aim of this study, therefore, was to investigate changes in lower body strength, jump and sprint performances across the English county cricket season. Methods: Male cricketers $(n=12$; age $24.4 \pm 2.3$ years; body mass, $84.3 \pm 9.9 \mathrm{~kg}$; height, $184.1 \pm 8.1 \mathrm{~cm})$ performed countermovement jumps $(\mathrm{CMJ})$ and $20 \mathrm{~m}$ sprints on 4 separate occasions, and back squat strength testing on 3 separate occasions across a competitive season. Results: Both absolute $(12.9 \%, P=0.005$, effect size $(\mathrm{ES})=0.53)$ and relative lower body strength $(15.8 \%, P=0.004, \mathrm{ES}=0.69)$ and $\mathrm{CMJ}$ height $(5.3 \%, P=0.037, \mathrm{ES}=0.42)$ improved significantly over the pre-season training period, although no significant change $(1.7 \%, P>0.05)$ in sprint performance was observed. Contrastingly, absolute $(14.3 \%, P=$ $0.001, \mathrm{ES}=0.72)$ and relative strength $(15.0 \%, P=0.001, \mathrm{ES}=0.77), \mathrm{CMJ}$ height $(4.2 \%, P$ $=0.023, \mathrm{ES}=0.40)$ and sprint performance $(3.8 \%, P=0.012, \mathrm{ES}=0.94)$ declined significantly across the season. Conclusions: The results of this study show that both the demands of the competitive cricket season and current in-season training practices do not provide a sufficient stimulus to maintain strength, jump, and sprint performances in these cricketers. Therefore, coaches should implement a more frequent, higher load strength training program across the competitive cricket season.
\end{abstract}

Key words: competition, maintenance, performance, jump, sprint, squat 
100

101

102

103

104

105

106

107

108

109

110

111

112

113

114

115

116

117

118

119

120

121

122

123

124

125

126

127

128

129

130

131

132

133

134

135

136

137

138

139

140

141

142

143

144

145

146

147

148

149

\section{INTRODUCTION}

With the increasing popularity of Twenty-20 and one-day cricket, the intensity of the game has increased. Batsmen are increasingly expected to score more runs, which involves taking more risks and requires the ability to run faster between the wickets. Glazier et al. ${ }^{1}$ identified a strong correlation between run up speed and ball release speed $(r=0.70-0.73)$ of fastmedium bowlers, with other studies also reporting running speed as a predictor of ball release speed. ${ }^{2,3}$ Sprinting is often involved in moments that directly affect the outcome of the game, therefore high sprinting speed capacity is considered to be an important attribute of the modern cricketer. ${ }^{4}$

Studies investigating the relationship between strength and sprint performance have observed a moderate-strong significant correlations between the two in a variety of sports. ${ }^{5-8}$ In addition, Comfort et al. ${ }^{9}$ reported that $20 \mathrm{~m}$ sprint time $(3.03 \pm 0.09 \mathrm{~s}$ to $2.85 \pm 0.11 \mathrm{~s} ; P<$ $0.001)$ improved concurrently with one repetition maximum (1RM) back squat strength (1.78 $\pm 0.27 \mathrm{kgkg}^{-1}$ to $2.05 \pm 0.21 \mathrm{~kg} \cdot \mathrm{kg}^{-1} ; P<0.001$ ) over an 8 week training period undertaken by rugby league players. Similarly, research has also identified a strong relationship between vertical jump height and sprint time in numerous sports, ${ }^{6,8,10}$ with Carr et al. ${ }^{10}$ recently reporting a strong correlation between countermovement jump (CMJ) height and $20 \mathrm{~m}$ sprint times $(r=-0.74)$ in a group of first-class county cricketers and Foden et al. ${ }^{11}$ reporting similar findings between these variables in academy cricketers $(r=-0.67)$. It is, therefore, no surprise that strong associations have also been found between lower body strength and vertical jump height. Wisloff et al. ${ }^{8}$ reported a correlation of $r=0.78$ between 1RM half squat strength and CMJ height, while Comfort et al. ${ }^{6}$ reported a correlation of $r=0.76$ between maximum back squat strength and CMJ height. These relationships between strength, jump and sprint performance suggest that maintaining strength levels is vital in maintaining jump and sprint performance.

A combination of strength training and conditioning is typically performed throughout the pre-season period, preparing cricketers for the start of the season. Justifiably, and as in many skill-based sports, focus then shifts towards technical and tactical preparation as the competitive season approaches. However, this transition to technical and tactical work is often conducted at the expense of regular strength training and in some circumstances the cessation of strength training. Over the course of a 26 week competitive season, this oversight is worrying from both performance and injury prevention perspectives, as declines in strength are observed 2-4 weeks following the cessation of strength training. ${ }^{12}$ This notion may have negative implications for sprint and jump performance throughout the competitive season, given the aforementioned association observed between strength and sprint and jump performances. 6,8

The competitions themselves conducted throughout the in-season period can, in some sports, provide a sufficient stimulus to maintain or even improve strength and power levels. ${ }^{13-15}$ Hoffman et al. ${ }^{13}$ for example, suggested that the demands of the basketball season provided an adequate stimulus to maintain leg strength and vertical jump performance, although there was a slight decrease in performances in the middle of the season. Less physically demanding sports, such as cricket, may not allow this to happen, however, as games are unlikely to provide a sufficient strength and/or power stimulus.

With the large ratio of aerobic compared to strength or power based activities during one-day and test game play for both bowlers and fielders, ${ }^{16}$ cricket provides a challenge to improving 
muscle strength, power and speed during the competitive season. However, studies have effectively maintained, or improved, strength, power, and speed across a competitive season $^{17-19}$ by implementing in-season strength training programs. Baker ${ }^{20}$ reported that subelite rugby league players increased lower body performance, while elite athletes managed to maintain performance across the season. The protocol used by Marques et al. ${ }^{18}$ also involved variation in volume and intensity; however, cricket presents unique demands due to the long duration (e.g. four days) of competitive games, and relatively low intensities. ${ }^{16}$ More research, therefore, is required into in-season training strategies adopted by cricketers in order to identify and develop optimal strategies.

The aim of this study was to investigate the variation in strength, jump and sprint performance of English county cricketers across the pre-season period and the English county season. It was hypothesized that strength, jump and sprint performance would improve over the long off-season and pre-season training periods ( 20 weeks). It was also hypothesized that strength, jump and sprint performance would then decline throughout the competitive season, due to the reduction in the frequency and therefore overall volume of strength training.

\section{METHODS}

\section{Subjects}

All subjects were regular first team first-class county cricketers $(n=12$; age $24.4 \pm 2.3$ years; body mass $84.3 \pm 9.9 \mathrm{~kg}$; height $184.1 \pm 8.1 \mathrm{~cm})$ from the same club. Subjects consisted of all rounders $(n=6)$, batsmen $(n=4)$ and spin bowlers $(n=2)$. They were provided with full participant information and all provided written informed consent. The study protocol was approved by the institutional ethics committee and conformed to the principles of the World Medical Association's Declaration of Helsinki (1983). Players from the team that regularly missed strength training sessions due to injury or illness across the season were excluded from analysis, resulting in the sample size of 12 .

\section{Design}

This study used a repeated measures observational design to identify the changes in strength, power and speed of English county cricketers across the English county season. The sprint 20 $\mathrm{m}$ distances represented the short sprints performed when running between the wickets $(17.68$ $\mathrm{m})$, with CMJ height selected as an indicator of lower body power. The three repetition maximum (3RM) back squat test was selected as a measure of lower body strength. Testing was performed at the start of the off-season training period (week 1), at the end of pre-season (week 20), in-season (week 36), and at the end of the season (week 46). Strength testing was not performed for the mid-season testing (i.e. week 36) due to restrictions made by technical coaches due to the high volume of fixtures.

All subjects were instructed to arrive at each session as they would to training, in a fed and hydrated state, in an attempt to standardize the athletes' status prior to each testing session. None of the subjects were injured during the testing period. The subjects were familiar with all of the tests completed as they formed part of the normal monitoring at the cricket club. All speed and jump tests were performed on an indoor cricket surface which the subjects were accustomed to training and testing on. Subjects were from the same club, as in a previous study by Carr et al., ${ }^{10}$ in which they performed the jump and sprint tests incorporated in the current study. The $\mathrm{CMJ}$ and $20 \mathrm{~m}$ sprint tests were found to be reliable both within-session (ICC $=0.987$ and 0.964, respectively) and between-sessions $($ ICC $=0.966$ and 0.923, respectively). 


\section{Methodology}

The subjects performed the tests in the following order: CMJ, $20 \mathrm{~m}$ sprints, strength testing. Each testing session was conducted at the same time of day, $\geq 48$ hours after any previous training or competition. Testing was conducted in small groups to increase the level of competition and aid in the motivation of the players to aid in ensuring maximal effort.

\section{Jump Tests}

Prior to the CMJ, subjects undertook a standardized 5 minute non-fatiguing dynamic warmup, including mobilisation exercises and various jumping activities. All subjects performed 3 trials with 2 minutes recovery time between each trial. The best performance of the 3 trials was reported for comparison between testing sessions.

The subjects were required to keep their hands on their hips throughout each jump trial to eliminate the facilitative use of the arms. Jump height was assessed using a portable jump mat (Fit Tech, Australia), which calculated jump height from flight time. Flight time was defined as the period between the instants of take-off and subsequent ground contact upon landing. This time was then used in the equation of uniform acceleration (A) to determine jump height:

\section{Sprint Tests}

$$
J H=\frac{9.81 \times F T^{2}}{8}
$$

Where $J H=$ jump height and $F T=$ flight time

The subjects undertook a standardized 10 minute warm-up which included activation and mobilisation exercises in addition to sprint drills and progressive sprints. The subjects performed three sprints each, with 2.5 minutes rest between each trial. The time taken to run $20 \mathrm{~m}$ was measured using Brower timing gates (Draper, Utah, USA). The subjects started 0.5 $\mathrm{m}$ behind the first timing gate at $0 \mathrm{~m}$, using a two point stationary start. There was a $20 \mathrm{~m}$ run off after the final timing gate to reduce the possibility of the subjects decelerating early, with the lead investigator visually checking that each subject attempted to accelerate through the entire $20 \mathrm{~m}$. The best of three trials was reported for comparison between testing sessions.

\section{Strength Tests}

The subjects performed a standardized barbell warm-up which included squat and lunge variations. Subjects then performed three warm-up sets of 5, 3, and 2 reps at $50 \%, 75 \%$, and $90 \%$ of the target load, respectively. They then performed a 3RM back squat set, with 1RM back squat performance subsequently predicted using the Brzycki equation. ${ }^{21}$ If the subject exceeded 3 repetitions they rested for 3-5 minutes before repeated the set at a heavier load, with increments of 2.5-5.0 kg dependent on the individual's previous performance. Whilst this method is an estimation of maximal strength calculated using a regression, it has been shown to be an accurate method of predicting 1RM back squat performance. ${ }^{22}$ The $3 \mathrm{RM}$ back squat protocol was selected to reduce the risk of musculoskeletal injury, particularly as the subjects did not perform regular maximal strength training. Predicted 1RM values were then calculated and expressed as relative measures (predicted 1RM / body mass) to take into account any changes in body mass across the season. 
Strength training programmes were split into phases (Tables 1-4), with the repetition volumes designed as a range, depending on the players' role and training age. Strength training sessions were performed twice per week during the off-season period (weeks 1-14), then once per week during pre-season (weeks 15-20) and the competitive season (week 21 onwards). The in-season strength programme (Table 4) was performed from week 19, except for week 20, when the session was replaced by strength testing (T2). However, adherence to the programme declined noticeably from week 24 . Training frequency was one session per week (100\% adherence) until week 24 , then approximately one session per month $(25 \%$ adherence rate) between weeks 24 and 46. Due to lack of adherence to the program, program content remained unchanged between weeks 24 and 46.

Subjects also performed a small volume of sprint technique training integrated into their warm ups prior to skill based training. Additionally 20 minutes of maximal aerobic speed (MAS) training at 110-120\% MAS was conducted once per week prior to a skill based training sessions, across the duration of the study.

\section{**INSERT TABLES 1-4 ABOUT HERE**}

\section{Statistical Analyses}

Normal distribution was assessed using Shapiro-Wilk's test of normality. Repeated measures analysis of variance (RMANOVA), with Tukey least significant difference (LSD) post-hoc analysis used to determine differences in CMJ and strength data across time points. Friedman's test, with multiple Wilcoxon signed ranks tests and Bonferroni correction, was performed to compare sprint performances across time points. SPSS software (version 20.0, IBM) was used in all of the above calculations. Data is presented as percentage change including 90\% confidence intervals (CI). Additionally, effect sizes (ES) were calculated using Cohen's d and interpreted by the criteria proposed by Rhea. ${ }^{23}$ The subjects in this study were considered as recreationally trained as they had been training consistently for between 1 and 5 years and demonstrated low relative strength levels, therefore effect sizes were interpreted as follows; large as $>1.5$, moderate as $0.80-1.50$, small as $0.35-0.80$, and trivial as $<0.35$.

\section{RESULTS}

There were no significant $(P>0.05)$ changes in body mass across the season (Table 5). CMJ performance decreased significantly $(P<0.001$, Power $=0.87)$ across time points with Tukey's LSD pairwise comparison revealing a small yet significant improvement between T1 and $\mathrm{T} 2(5.2 \%, 90 \% \mathrm{CI}=4.25-6.15, P=0.037, \mathrm{ES}=0.42)$ and a small but significant decline between T2 and T4 $(4.3 \%, 90 \% \mathrm{CI}=3.22-5.30, P=0.023, \mathrm{ES}=0.40)$ (Table 5). Sphericity was assumed via Mauchley's test $(P>0.05)$.

**INSERT TABLE 5 ABOUT HERE** 
Sprint performances decreased significantly $(P<0.001$, Power $=0.82)$ between testing sessions, with Wilcoxon's test revealing small to moderate and significant declines in performance between T2 and T3 $(2.3 \%, 90 \% \mathrm{CI}=1.67-2.99, P=0.024, \mathrm{ES}=0.61)$, and T2 and T4 $(4.0 \%, 90 \% \mathrm{CI}=2.93-5.07, P=0.012, \mathrm{ES}=0.94)($ Table 5$)$.

Lower body strength (3RM back squat) changed significantly $(P<0.001$, Power $=0.99)$ between testing sessions, with Bonferroni post-hoc analysis showing a small but significant increase occurred between T1 and T2 $(12.9 \%, 90 \% \mathrm{CI}=9.91-15.89, P=0.005, \mathrm{ES}=0.53)$, and a small yet significant decrease between T2 and T4 $(14.2 \%, 90 \% \mathrm{CI}=11.16-17.24, P=$ $0.001, \mathrm{ES}=0.72$ ) (Table 5). Similarly relative strength (predicted $1 \mathrm{RM} /$ body mass) demonstrated significant differences $(P<0.001$, Power $=0.99)$ between testing sessions, with Bonferroni post-hoc analysis identifying a small but significant improvement between $\mathrm{T} 1$ and T2 $(15.7 \%, 90 \% \mathrm{CI}=12.47-18.93, P=0.004, \mathrm{ES}=0.69)$, and a small yet significant decline between T2 and T4 $(15.0 \%, 90 \% \mathrm{CI}=12.01-17.99, P=0.001, \mathrm{ES}=0.77)$ (Table 5).

\section{DISCUSSION}

As hypothesized, strength, jump and sprint performances all improved between the start of the off-season and end of the pre-season period (T1-T2) (Table 5), although the small improvement in sprint performance was not statistically significant $(P>0.05)$. Additionally, as hypothesized, 3RM strength $(14.2 \%, 90 \% \mathrm{CI}=11.16-17.24 \%)$, relative strength $(15.0 \%$, $90 \% \mathrm{CI}=12.01-17.99 \%)$, CMJ height $(4.3 \%, 90 \% \mathrm{CI}=3.22-5.30 \%)$, and sprint $(4.0 \%, 90 \%$ $\mathrm{CI}=2.93-5.07 \%)$ performances subsequently declined between the end of pre-season and the end of the competitive season (T2-T4) (Table 5). Body mass did not differ significantly across the season and therefore is unlikely to have influenced any of the performance variables.

A study with a similar protocol investigated the effect of detraining in the handball season. ${ }^{17}$ Subjects performed resistance training during pre-season for a period of 12 weeks, over which sprint performance, loaded and unloaded CMJ height, and ball throw speed improved. Resistance training was then discontinued for a period of 7 weeks. The authors reported no significant decline in CMJ or sprint performance, but a significant decline in ball throw speed. The reason for no significant decline in sprint and CMJ performance could have been due to the volume of sprinting and jumping performed during competitive play in handball which may have served as a sufficient in-season force and power stimulus for maintaining sprint and jump performances for this cohort. Additionally, this detraining period was only 7 weeks in length and so a significant decline may have been observed over a longer detraining period, similar to that of the current study which saw a decline in CMJ performance after 16 weeks of the competitive season.

Resistance training performed over a competitive lacrosse season ( 24 weeks) of similar length as the current study (26 weeks) elicited improvements in sprint and change of direction performance. ${ }^{24}$ The subjects had similar anthropometric and strength characteristics to those in the present study, therefore, had resistance training continued over the course of the county cricket season, similar findings may have been observed. However, differences in resistance training between groups may have affected the results. Another factor to be considered is that in the study by Thomas et $a .^{24}$ competitive matches occurred on one day per week 
348 throughout the study period, rather than the five days in the current study, allowing more time 349 to perform non-game specific training.

Researchers have shown that the session design of in-season resistance training is also important to ensure maintenance or development of specific athletic attributes. ${ }^{18,25-28}$ Studies have observed that prolonged periods of training at low-moderate intensity do not prevent a decline in strength levels. ${ }^{25}$ Training at low volumes for a prolonged period may trigger a decrease in lean body mass, power, and speed. ${ }^{20}$ This is supported by the findings of the current study. Maintaining the intensity of strength training is also essential in the maintenance or development of strength and with the underlying influence of strength on sprint and jump performance, this must be considered when designing an in-season programme. Moreover, conclusions of a meta-analysis were that the optimum intensity for maximal strength gains is $85 \% 1 \mathrm{RM}^{29}$

Training at half the volume and frequency of pre-season training, but maintaining $80-90 \%$ $1 \mathrm{RM}$ intensity was enough to maintain strength at near pre-season levels for 10 weeks across an American football season. ${ }^{26}$ Sprint and vertical jump performance also improved. This suggests that the in-season programme in the current study may have been effective if adhered to. However, one methodological concern with this study was the very short preseason period of only 2.5 weeks. ${ }^{26}$ This, and the community college standard of the athletes, means their capacity for adaptation may be much greater than athletes with a longer training history and a longer pre-season period. Collegiate athletes typically participate in other sports and so the college-level athletes observed in the aforementioned study may well have been training for another sport which may have also contributed to the observed performance results.

Research has shown the importance of variation in training stimuli. Studies investigating the effect of high force and high power resistance training methods on strength and power levels suggest that both methods can be ineffective when used alone and over a prolonged period. ${ }^{27}$, 28,30 Newton et al. ${ }^{28}$ observed no improvements in jump height in elite volleyball subjects after performing heavy slow resistance training over the pre-season period. The same research group found that the addition of ballistic training stopped the decline in jump performance which occurred whilst performing exclusively heavy slow resistance training. However, performing explosive resistance training exclusively was not sufficient to maintain maximum and explosive strength in female volleyball players. ${ }^{27}$

Other research has shown that a varied program may be most effective training method. ${ }^{30,31}$ Harris et $a l .{ }^{30}$ observed greater benefits from using a combined programme than high force or high power programmes in three groups of previously trained men. Marques et al. ${ }^{18}$ observed an improvement in lower body strength and CMJ height over a 12 week volleyball season, with session volumes and intensities varied between 3 sets of 3-6 repetitions at 50-80\% 4RM. These sessions included loaded jumping drills that the subjects had not previously performed, which may account for the improved performances. Whether this intensity is sufficient to be the cause for the observed improvements is questionable. The training effect may have derived from the combination of moderate intensity resistance training and plyometric training, or the novel training stimulus provided by the loaded jumping exercises.

This type of programme provides a simple way to introduce variation into athletes' programmes. With regard to its potential application to cricket, it must be taken into consideration that this programme was designed for rugby which requires maintenance of 
hypertrophy over the competitive season. Whilst the variation in this protocol could be useful for cricket athletes, hypertrophy, particularly in the upper body of fast bowlers, may not be beneficial or, therefore, desired. ${ }^{32}$ Keeping repetitions lower and maintaining intensity has been shown to elicit strength gains with less hypertrophy. ${ }^{33-35}$

Due to the unique demands and fixture scheduling in cricket, further research should be conducted investigating the efficacy of varied in-season strength training protocols. Research in basketball has shown that a training frequency of two sessions per week is effective at maintaining strength levels for up to 6 months, ${ }^{36}$ with research in other sports showing similar findings. ${ }^{20,}{ }^{24}$ However, one session per week has been shown to be effective at preventing a significant decline in strength, sprint and jump performance over 12 weeks if the intensity of the session is equal to at least 4RM ( 90\% 1RM). ${ }^{19}$ It is difficult to determine whether these findings would occur over a longer period, therefore, training frequency may be a key factor to investigate further, given the limited time opportunities available during the competitive cricket season.

\section{PRACTICAL APPLICATIONS}

The results of this study show that the physical demands of the English county cricket season alone are not enough to maintain pre-season strength, jump and sprint performance. Findings from research in a number of other sports show that performing regular resistance training can not only maintain, but improve pre-season levels of strength across a competitive season. Coaches should implement a time-effective resistance training strategy in-season, adopting a varied wave-like periodization. Based on research findings, programmes should maintain a minimum intensity ( $\geq 80 \% 1 \mathrm{RM}$ ) usually associated with strength training and a minimum frequency of one session per week, but ideally two sessions week, depending on the competition schedule.

\section{CONCLUSIONS}

Both the demands of the competitive English county cricket season and current in-season training practices undertaken by these county cricketers do not provide a sufficient stimulus to maintain pre-season levels of strength, jump and sprint performance across this period. It is therefore suggested that county cricket players include $\geq 1$ strength training session per week, incorporating compound movements at loads $\geq 80 \% 1 \mathrm{RM}$. 


\section{REFERENCES}

1. Glazier PS, Paradisis GP, Cooper SM. Anthropometric and kinematic influences on release speed in men's fast-medium bowling. J Sports Sci. Dec 2000;18(12):10131021.

2. Duffield R, Carney M, Karppinen S. Physiological responses and bowling performance during repeated spells of medium-fast bowling. J Sports Sci. Jan 1 2009;27(1):27-35.

3. Salter CW, Sinclair PJ, Portus MR. The associations between fast bowling technique and ball release speed: a pilot study of the within-bowler and between-bowler approaches. J Sports Sci. Sep 2007;25(11):1279-1285.

4. Petersen CJ, Pyne D, Dawson B, Portus M, Kellett A. Movement patterns in cricket vary by both position and game format. J Sports Sci. Jan 2010;28(1):45-52.

5. Comfort P, Bullock N, Pearson SJ. A comparison of maximal squat strength and 5-, 10 -, and 20-meter sprint times, in athletes and recreationally trained men. J Strength Cond Res. Apr 2012;26(4):937-940.

6. Comfort P, Stewart A, Bloom L, Clarkson B. Relationships Between Strength, Sprint, and Jump Performance in Well-Trained Youth Soccer Players. The Journal of Strength \& Conditioning Research. 2014;28(1):173-177.

7. Kirkpatrick J, Comfort P. Strength, Power, and Speed Qualities in English Junior Elite Rugby League Players. The Journal of Strength \& Conditioning Research. 2012;27(9):2414-2419

8. Wisloff U, Castagna C, Helgerud J, Jones R, Hoff J. Strong correlation of maximal squat strength with sprint performance and vertical jump height in elite soccer players. Br J Sports Med. Jun 2004;38(3):285-288.

9. Comfort P, Haigh A, Matthews MJ. Are Changes in Maximal Squat Strength During Preseason Training Reflected in Changes in Sprint Performance in Rugby League Players? J Strength Cond Res. Feb 3 2012;26(3):772-776.

10. Carr C, McMahon JJ, Comfort P. Relationships between jump and sprint performance in first-class county cricketers. Journal of Trainology. 2015;4(1):1-5.

11. Foden M, Astley S, Comfort P, McMahon JJ, Matthews MJ, Jones PA. Relationships between speed, change of direction and jump performance with cricket specific speed tests in male academy cricketers. Journal of Trainology. 2015;4(2):37-42.

12. Mujika I, Padilla S. Detraining: loss of training-induced physiological and performance adaptations. Part I: short term insufficient training stimulus. Sports Med. Aug 2000;30(2):79-87.

13. Hoffman JR, Fry AC, Howard R, Maresh CM, Kraemer WJ. Strength, Speed and Endurance Changes During the Course of a Division I Basketball Season. The Journal of Strength \& Conditioning Research. 1991;5(3):144-149.

14. Thomas V, Reilly T. Changes in fitness profiles during a season of track and field training and competition. Br J Sports Med. Dec 1976;10(4):217-222.

15. Thomas V, Reilly T. Fitness assessment of English league soccer players through the competitive season. Br J Sports Med. Sep 1979;13(3):103-109.

16. Petersen CJ, Pyne DB, Portus MR, Dawson BT. Comparison of Player Movement Patterns Between 1-Day and Test Cricket. The Journal of Strength \& Conditioning Research. 2011;25(5):1368-1373.

17. Marques MC, Gonzalez-Badillo JJ. In-season resistance training and detraining in professional team handball players. J Strength Cond Res. Aug 2006;20(3):563-571. 
498 18. Marques MC, Tillaar R, Vescovi JD, Gonzalez-Badillo JJ. Changes in strength and power performance in elite senior female professional volleyball players during the in-season: a case study. J Strength Cond Res. Jul 2008;22(4):1147-1155.

19. Ronnestad BR, Nymark BS, Raastad T. Effects of in-season strength maintenance training frequency in professional soccer players. $J$ Strength Cond Res. Oct 2011;25(10):2653-2660.

20. Baker D. The effects of an in-season of concurrent training on the maintenance of maximal strength and power in professional and college-aged rugby league football players. J Strength Cond Res. May 2001;15(2):172-177.

21. Brzycki M. Predicting a one-rep max from reps to failure. $J$ Health Phys Ed Rec and Dance. 1993;64:88-90.

22. LeSeur D, McCormick J, Mayhew J, Wasserstein R, Arnold M. The accuracy of prediction equations for estimating 1-RM performance in bench press, squat and deadlift. J Strength Cond Res. 1997;11:211-213.

23. Rhea MR. Determining the Magnitude of Treatment Effects in Strength Training Research Through the Use of the Effect Size. The Journal of Strength \& Conditioning Research. 2004;18(4):918-920.

24. Thomas C, Mather D, Comfort P. Changes in sprint, change of direction and jump performance during a competetive season in male lacrosse players. Journal of Athletic Enhancement. 2014;3(5):53-60.

25. Caterisano A, Patrick BT, Edenfield WL, Batson MJ. The Effects of a Basketball Season on Aerobic and Strength Parameters Among College Men: Starters vs. Reserves. The Journal of Strength \& Conditioning Research. 1997;11(1):21-24.

26. dos Remedios KA, dos Remedios RL, Loy SF, et al. Physiological and Field Test Performance Changes of Community College Football Players Over a Season. The Journal of Strength \& Conditioning Research. 1995;9(4):211-215.

27. Hakkinen K. Changes in physical fitness profile in female volleyball players during the competitive season. J Sports Med Phys Fitness. Sep 1993;33(3):223-232.

28. Newton RU, Kraemer WJ, Hakkinen K. Effects of ballistic training on preseason preparation of elite volleyball players. Med Sci Sports Exerc. Feb 1999;31(2):323330.

29. Peterson MD, Rhea MR, Alvar BA. Maximizing strength development in athletes: a meta-analysis to determine the dose-response relationship. J Strength Cond Res. May 2004;18(2):377-382.

30. Harris GR, Stone M, O'Bryant HS, Proulx CM, Johnson R. Short-term performance effects of high power, high force or combined weight training methods. J Strength Cond Res. 2000;14(1):14-20.

31. Hakkinen $\mathrm{K}$, Alen $\mathrm{M}$, Kraemer WJ, et al. Neuromuscular adaptations during concurrent strength and endurance training versus strength training. Eur J Appl Physiol. Mar 2003;89(1):42-52.

32. Stronach BJ, Cronin JB, Portus MR. Part 2: Mechanical and Anthropometric Factors of Fast Bowling for Cricket, and Implications for Strength and Conditioning. Strength \& Conditioning Journal. 2014;36(5):53-60.

33. Kraemer WJ, Marchitelli L, Gordon SE, et al. Hormonal and growth factor responses to heavy resistance exercise protocols. J Appl Physiol (1985). Oct 1990;69(4):14421450.

34. Schoenfeld BJ, Peterson MD, Ogborn D, Contreras B, Sonmez GT. Effects of LowVersus High-Load Resistance Training on Muscle Strength and Hypertrophy in WellTrained Men. The Journal of Strength \& Conditioning Research. 2015;Publish Ahead of Print. 
35. Schoenfeld BJ, Ratamess NA, Peterson MD, Contreras B, Sonmez GT, Alvar BA. Effects of Different Volume-Equated Resistance Training Loading Strategies on Muscular Adaptations in Well-Trained Men. The Journal of Strength \& Conditioning Research. 2014;28(10):2909-2918.

36. Hoffman JR, Maresh CM, Armstrong LE, Kraemer WJ. Effects of off-season and inseason resistance training programs on a collegiate male basketball team. Journal of Human Muscle Performance. 1991;1(1):48-55. 
598

599

600

601

602

603

604

605

606

607

608

609

610

611

612

613

614

615

616

617

618

619

620

621

622

623

624

625

626

627

628

629

630

631

632

633

634

635

636

637

638

639

640

641

642

643

644

645

646

647

648
Tables

Table 1. Example of training program during the General Preparation Phase 1 (Weeks 1-6)

\begin{tabular}{|l|c|l|c|}
\hline Session 1 & Sets x Reps & Session 2 & Sets x Reps \\
\hline \multicolumn{2}{|c|}{$60-70 \%$ 1RM } & \multicolumn{2}{|c|}{$60-70 \%$ 1RM } \\
\hline Back Squat & $3 \times 6-10$ & Overhead Squat & $3 \times 6-8$ \\
Romanian Deadlift & $3 \times 6-10$ & Mid-Thigh Clean Pull & $3 \times 6-8$ \\
Hip Thrusts & $3 \times 6-10$ & Hip Thrusts & $3 \times 6-10$ \\
Close Grip Pull Ups & $3 \times 8-10$ & Wide Grip Pull Ups & $3 \times 8-10$ \\
Weighted Press Ups & $3 \times 6-8$ & Behind Neck Press & $3 \times 5-8$ \\
\hline
\end{tabular}


Table 2. Example of training program during General Preparation Phase 2 (Weeks 7-14)

\begin{tabular}{|l|c|l|c|}
\hline Session 1 & Sets x Reps & Session 2 & \multicolumn{1}{|c|}{ S5-80\% 1RM } \\
\hline \multicolumn{1}{|c|}{$80-85 \%$ 1RM } & \multicolumn{1}{|c|}{} \\
\hline Back Squat & $3 \times 5-8$ & Overhead Squat & $3 \times 5-6$ \\
Romanian Deadlift & $3 \times 5-8$ & Mid-Thigh Power Clean & $3 \times 4-5$ \\
Hip Thrusts & $3 \times 5-8$ & Hip Thrusts & $3 \times 5-8$ \\
Close Grip Pull Ups (Weighted) & $3 \times 4-6$ & Prone Bench Pull & $3 \times 5-6$ \\
Weighted Press Ups & $3 \times 4-6$ & Push Press & $3 \times 4-6$ \\
\hline
\end{tabular}




\begin{tabular}{|l|c|}
\hline Session 1 & Sets x Reps \\
\hline \multicolumn{2}{|c|}{ 60-80\% 1RM } \\
\hline Power Clean & $3 \times 4-5$ \\
Back Squat & $3 \times 5-6$ \\
Hip Thrusts & $3 \times 4-6$ \\
Romanian Deadlifts & $3 \times 5-6$ \\
Close Grip Pull Ups (Weighted) & $3 \times 4-5$ \\
\hline
\end{tabular}


Table 4. Example of in-season (Competition Phase) training program (Week 19 onward)

\begin{tabular}{|l|c|}
\hline Session 1 & Sets x Reps \\
\hline \multicolumn{2}{|c|}{$70-90 \%$ 1RM } \\
\hline Power Clean & $3 \times 3$ \\
Back Squat & $3 \times 3-5$ \\
Romanian Deadlifts & $3 \times 5$ \\
Close Grip Pull Ups (Weighted) & $3 \times 3$ \\
\hline
\end{tabular}

756

757

758

759

760

761

762

763

764

765

766

767

768

769

770

771

772

773

774

775

776

777

778

779

780

781

782

783

784

785

786

787 
Table 5. Descriptive statistics (means \pm standard deviations) for body mass, jump, sprint, and strength testing results across the testing period.

\begin{tabular}{|c|c|c|c|c|}
\hline & T1 & $\mathrm{T} 2$ & T3 & T4 \\
\hline Body Mass (kg) & $84.3 \pm 9.9$ & $84.0 \pm 9.3$ & $83.8 \pm 9.2$ & $83.6 \pm 9.5$ \\
\hline CMJ $(\mathrm{cm})$ & $42.3 \pm 5.9$ & $44.5 \pm 4.5^{*}$ & $43.5 \pm 4.2^{* *}$ & $42.6 \pm 5.0^{* * *}$ \\
\hline Sprint (s) & $3.06 \pm 0.15$ & $3.00 \pm 0.11$ & $3.07 \pm 0.12^{* \star}$ & $3.12 \pm 0.13^{\star * *}$ \\
\hline 3RM (kg) & $97.1 \pm 25.9$ & $109.6 \pm 21.5^{*}$ & & $94.0 \pm 21.6^{* \star *}$ \\
\hline 1RM (kg/kg) & $1.27 \pm 0.30$ & $1.47 \pm 0.28^{*}$ & & $1.25 \pm 0.29^{* * *}$ \\
\hline
\end{tabular}

